\title{
Magnetic resonance imaging of the breast: A clinical perspective
}

\author{
J Edge, MB BS, FRCS (Edin), BSC, MMed \\ I Boeddinghaus, MB ChB, MRCP (Lond), MD (Lond) \\ Christiaan Barnard Memorial Hospital, Cape Town
}

Corresponding author: J Edge (jmedge@mweb.co.za)

\begin{abstract}
The role of magnetic resonance imaging (MRI) in screening for breast cancer and its use after the diagnosis of breast cancer is discussed. The topic is enormous, with over 5000 papers published in the last 10 years. In this précis, we focused on articles that examine its clinical relevance. We did not look at economic factors.
\end{abstract}

S Afr J Rad 2012;16(2):61-64

\section{MRI and breast cancer screening}

In 2004, an article was published in the NEJM looking at the use of MRI screening in 1909 high-risk Dutch women. ${ }^{1}$ The screening process included clinical examination, mammogram and MRI. The mean age of the women was $40 ; 18.7 \%$ had a known mutation. The sensitivity and specificity for the 3 screening modalities in this young cohort are shown in Table 1. The authors concluded that MRI screening could detect breast cancer at an early stage in high-risk women.

The results are in keeping with other studies. ${ }^{2,3}$ The findings have consistently shown that high-risk young women (whose disease tends to be missed with mammography) benefit from the addition of an MRI.
The American Cancer Society has combined the findings to give clear recommendations (Table 2). ${ }^{4}$ It is our opinion that South Africa should accept the recommendations where possible.

Two points highlighted in the American Cancer Society guidelines that are often glossed over, bear repetition. The first is that, within the context of high-risk breast screening, MRI should be used as an adjunct to, and not as a replacement for, mammography, given the lack of sensitivity of MRI for ductal carcinoma in situ (DCIS), and the tendency of the different modalities to diagnose cancers missed on the other. The second refers to the low positive predictive value (PPV) of MRI

Table 1. Adapted from data in the NEJM ${ }^{1}$

\begin{tabular}{llll}
\hline & $\begin{array}{l}\text { Clinical } \\
\text { examination } \\
(\%)\end{array}$ & $\begin{array}{l}\text { Mammography } \\
(\%)\end{array}$ & $\begin{array}{l}\text { MRI } \\
(\%)\end{array}$ \\
\hline $\begin{array}{l}\text { Sensitivity } \\
\text { (Invasive Ca and DCIS) }\end{array}$ & 17.8 & 40.0 & 71.1 \\
$\begin{array}{l}\text { Specificity } \\
\text { (Invasive Ca) }\end{array}$ & 98.1 & 95.0 & 90.0
\end{tabular}

Table 2. American Cancer Society Guidelines for Breast Screening with MRI as an adjunct to mammography, 2007

Recommend annual MRI screening as an adjunct to mammography (based on evidence)

Recommend annual MRI screening as an adjunct to mammography (based on expert consensus opinion)

Insufficient evidence to recommend for or against MRI screening
BRCA mutation

First degree relative of BRCA carrier but untested

Life risk $>20-25 \%$ as defined by model dependent on family history

Radiation to chest age 10 - 30

Li-Fraumeni (p53 gene mutation) syndrome and 1st-degree relatives

Cowden and Bannayan-Riley-Ruvalcaba (PTEN gene mutation) syndromes and 1st-degree relatives

Lifetime risk 15 - $20 \% \%$ as defined by model dependent on family history

LCIS $^{*}$ or atypical lobular hyperplasia

Atypical ductal hyperplasia

Extremely dense breasts on mammography

Women with a personal history of breast cancer including DCIS

Women $<15 \%$ lifetime risk 
(20 - 40\%), leading to a high rate of biopsies and further investigations - acceptable in women with a high risk of breast cancer, but potentially harmful to women in lower-risk categories.

It is our opinion that women who present with an unknown primary (e.g. with malignant axillary nodes) should have an MRI, in addition to conventional radiology, as this investigation has demonstrated high sensitivity for the detection of an ipsilateral breast cancer primary. ${ }^{5}$

\section{MRI in women with a proven breast cancer}

While it is clear that a small group of women will benefit from a screening MRI, its use in patients with a diagnosed breast cancer is less clear. Undoubtedly, the lesion is seen more clearly. Breast MRI post diagnosis has become a common pre-operative investigation, and the questions that need to be considered are whether its increased use is translating into clearer margins at the time of the initial surgery, decreased recurrence and, as a consequence, improved patient survival. Are patients benefitting or are they just having more radical surgery?

The comparative effectiveness of MRI in breast cancer (COMICE) trial was a multicentre trial based in the UK. ${ }^{6}$ Forty-five breast centres took part in a prospective randomised study. Over 1600 women who had been diagnosed with breast cancer and selected for breast conservation therapy (BCT) were enrolled. They were randomised into 2 groups: half received an MRI pre-operatively, and half did not. The outcomes measured were the percentage of patients who underwent re-excision or mastectomy within 6 months of their initial surgery and also the number of patients who had an avoidable mastectomy. The surgical margins had to be clear after their initial surgery. The investigators did not consider the question of survival. The patients were well matched pathologically and demographically. The results are shown in Table 3. As can be seen, there was no difference in re-excision rate, and pre-operative MRI resulted in a statistically non-significant decrease in the number of completion mastectomies.

As a result of a pre-operative MRI, 7\% of patients were advised to have a mastectomy rather than BCT. In retrospect, $30 \%$ of these mastectomies were unnecessary (the MRI over-estimated the size of the tumour (see Case 1) or there was no pathological evidence of multicentricity as indicated by the scan). One patient had an undetected lung metastasis. Two per cent had contra-lateral procedures following the MRI, and $4 \%$ had biopsies.

Based on the trial's findings, the PPV of an MRI scan was calculated to be $62 \%$, and the negative predictive value of $84 \%$ (Case 2) illustrates the problem of a low PPV. The number of lobular cancers in the study group was considered to be too low to allow any definitive guidelines.

\section{Table 3. Outcome from the COMICE trial: results adapted}

\begin{tabular}{lll}
\hline & MRI & No MRI \\
\hline Time to surgery (days) & 14 & 13 \\
BCT (\%) & 92 & 95 \\
Mastectomy (\%) & 7 & $\begin{array}{l}1 \text { (patient } \\
\text { choice) }\end{array}$ \\
Re-excision (\%) & 10 & 11 \\
Completion mastectomy (\%) & 6 & 8 \\
Pathologically avoidable mastectomy (\%) & 2 & $<1$
\end{tabular}

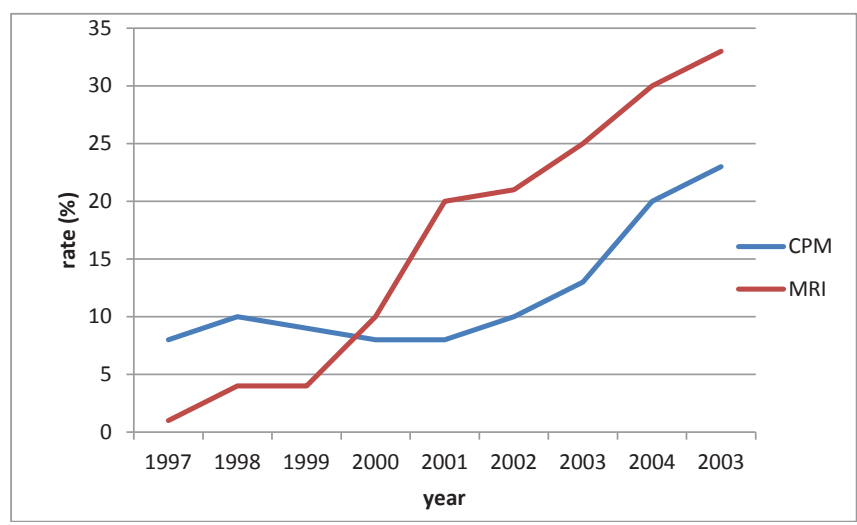

Fig. 1. The relationship between MRI usage and CPM rates and use of MRI at diagnosis by year of surgery (based on data from King et al.) ${ }^{14}$

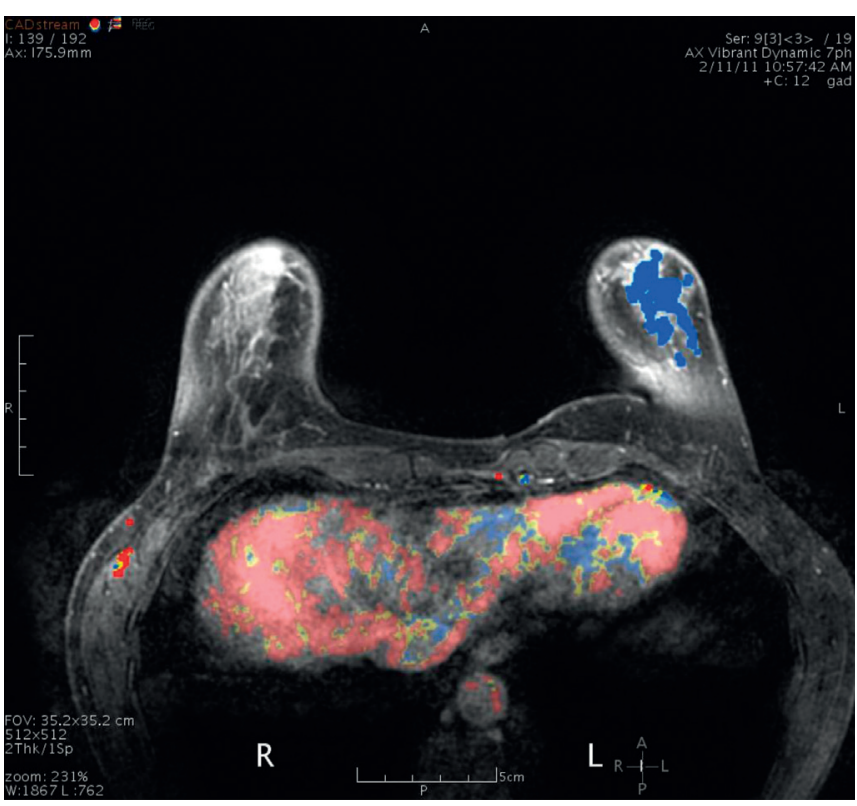

Fig. 2. The MRI over-estimated the size of the lesions. The patient had appropriate surgery.

The conclusions from the COMICE trial were that $(i)$ the rate of reoperation for BCT was unchanged by pre-operative MRI, and (ii) that all MRI lesions seen must be biopsied.

Other centres have noted an increase in the mastectomy rate for early breast cancer. ${ }^{7}$ This is a reversal of the trends seen in the 1990s. In 1990, the NIH recommended BCT as the preferred treatment for early breast cancer. ${ }^{8}$ In $1989,35 \%$ of women with early breast cancer in the USA were treated with BCT. This figure rose to $60 \%$ in $1995 .{ }^{9}$ Numerous studies have shown that the outcome in the two groups was equivocal and that the patients who had BCT did better from a psychosocial perspective. $^{10}$

The incidence of local recurrence has decreased over the last 20 years. The incidence of $8 \%$ local recurrence for stage 1 disease seen in the NSBAP trial has reduced to as little as $1.8 \%$ in some centres with the improvement of adjuvant therapy ${ }^{11}$ MRI studies have quoted the change in surgical management based on MRI findings for between 8 and $30 \%$ of all patients. ${ }^{6,12}$ Local recurrence data show that most of these patients would not have needed their mastectomy in retrospect. Radiotherapy, together with the added benefit of improved systemic 
adjuvant treatment, has proved to be very effective in dealing with small residual areas of disease within the breast.

Few papers have looked at whether the outcome of patients with breast cancer has improved since MRI usage became more commonplace. A retrospective study by Solin et al. reviewed 756 women who were treated with $B C T^{13}$ where 215 had an MRI scan pre-operatively and 541 did not. The study was not randomised but the groups were treated concurrently. The timing of the MRI was variable (27\% before the core biopsy, $23 \%$ after the core biopsy, $37 \%$ after the initial excision and $6 \%$ after re-excision). Although the MRI group had slightly better pathological features, their outcome at 8 years was no different in terms of overall survival, cause-specific survival, distant metastases, local failure and the occurrence of contralateral breast cancer. This paper demonstrates the very real difficulties of detecting a breast cancer specific outcome in unselected patients, in an era where recurrence rates are low.

The usage of MRI scanning has also increased the rate of contralateral prophylactic mastectomy (CPM). A retrospective study by King et al. ${ }^{14}$ noted that there was an increase in CPM in the USA of $150 \%$ since 1998. In his paper, he noted that whilst the number of very high-risk patients (e.g. BRCA positive, mantle radiotherapy) remained constant, the general incidence of contralateral breast cancer had decreased. This is in part attributable to improved systemic therapies, where 5 years of adjuvant hormone therapy decreases CBC by $50-55 \%$, and chemotherapy by approximately $20 \% \cdot{ }^{15}$ The recent surveillance, epidemiology and end results (SEER) data from 1992 to 2004 estimate an annual risk of $0.1 \%$ in women aged $<50$ years.

King found the decision for a CPM was based on race, BRCA mutations, past history of mantle radiotherapy, the surgeon, MRI usage and non-invasive histology. The pre-operative usage of MRI in patients diagnosed with breast cancer increased from $1.3 \%$ to $36.3 \%$ over the same period. Forty-three per cent of patients choosing to have a CPM had had a pre-operative MRI as opposed to $16 \%$ in the patients choosing unilateral surgery. The relationship between MRI usage and CPM can be seen graphically in Fig. 1. In their single-institution study, at a median follow-up of 4.4 years for patients undergoing CPM and 6.8 years for patients not undergoing CPM, multivariate Cox regression demonstrated no difference in subsequent breast cancer event rates between the groups $(p=0.23$ ), and that prognosis was determined by the index lesions.

In conclusion, MRI scanning has a clearly defined role in breast screening. It is reasonable to adopt the American Cancer Society guidelines to guide us in South Africa. Its routine use for patients with a newly diagnosed breast cancer cannot be justified. There is no evidence that it decreases the re-excision or the recurrence rate following BCT, and it increases the number of mastectomies being performed. The available data suggest that the change in management of patients following an MRI will not increase their survival.

\section{Case 1}

A 40-year-old woman presented with a mass in her left breast. Clinically the mass was consistent with a benign process. Her mammogram was unhelpful and her sonar showed 3 discreet similarlooking masses; the largest was noted to be $11 \mathrm{~mm}$. Tru-cut of the palpable lesion confirmed the diagnosis of lobular carcinoma. FNA of the other lesions was not diagnostic. MRI scan showed a single large lesion $87 \times 44 \mathrm{~mm}$. On the basis of the MRI findings, the patient had

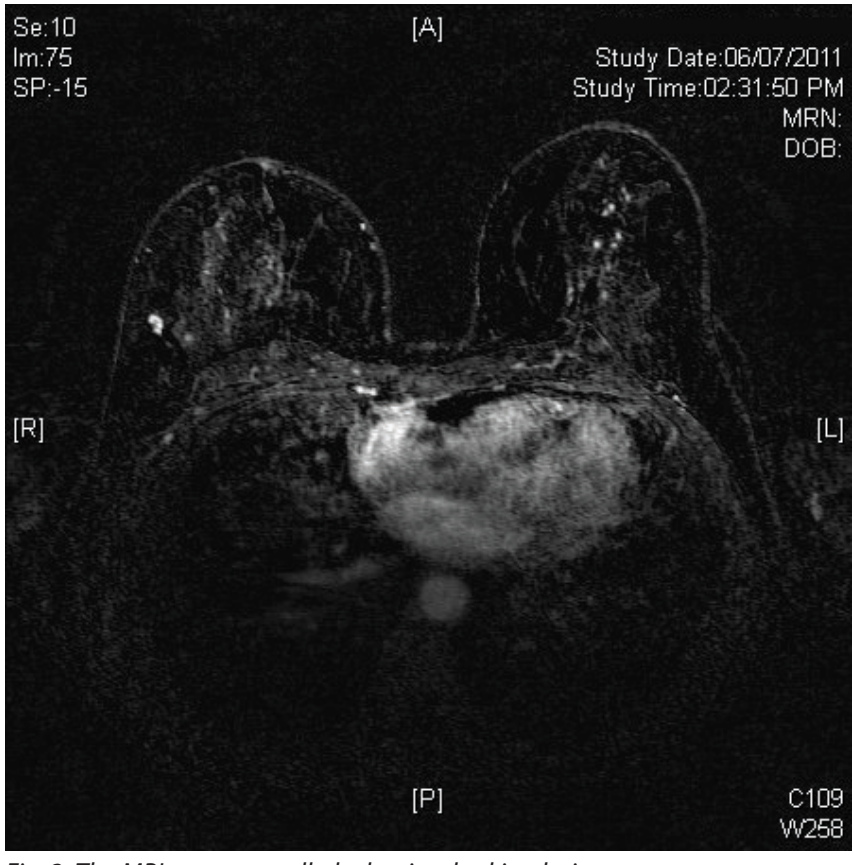

Fig. 3. The MRI scan overcalled a benign-looking lesion.

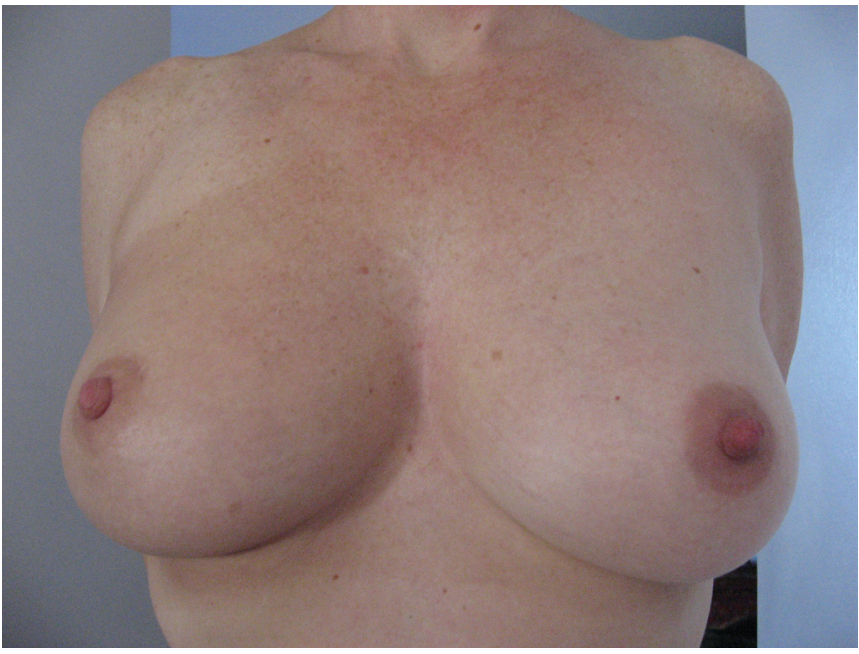

Fig. 4. The patient's cosmetic result.

a mastectomy. Her final histology showed 3 discreet lesions, which corresponded to those seen on ultrasound: $12 \mathrm{~mm}, 8 \mathrm{~mm}$ and $10 \mathrm{~mm}$ lobular carcinoma (Fig. 2).

\section{Case 2}

A 49-year-old woman was diagnosed with breast cancer in August 2010. Her mammogram showed a single malignant-looking lesion. Ultrasound noted 2 malignant-looking lesions and a further benignlooking lesion in the upper outer quadrant of her right breast. Tru-cut biopsy of one (malignant-looking) lesion confirmed the diagnosis of an infiltrating ductal carcinoma. FNA of the second malignant-looking lesion showed atypia. The third (benign-looking) lesion showed features in keeping with a benign proliferative mass. The patient did not want a mastectomy, so had a wide local excision of the 2 adjacent cancers and biopsy of the third lesion (ultrasound guided). The 2 breast cancers ( 3 $\mathrm{mm}$ and $4 \mathrm{~mm}$ ) were completely excised. The third lesion was noted 
to be a fibroadenoma. The patient had postoperative radiotherapy and hormone therapy.

In August 2011 the patient presented with a palpable mass in the upper outer quadrant of her right breast. Clinically the mass was suspicious. FNA of the mass was consistent with an intramammary lymph node. Her mammogram was normal, sonar showed a separate area of fat necrosis (aspirated) and the patient had an MRI that showed a suspicious lesion (BIRADS 4) in keeping with a new primary (Fig. 3). It was not clear whether this corresponded to the palpable lesion or sonographic lesion. The patient was happy with her cosmetic result (Fig. 4) so did not want to consider mastectomy. A clip was placed and the area was removed surgically. The lesion was shown to be a benign intramammary lymph node.

1. Kriege M, Brekelsmans CT, Boetes C, et al. Efficacy of MRI and mammography for breast cancer screening in women with a familial or genetic disposition. N Engl J Med 2004;351:427-437.

2. Leach MO, Boggis CR, Dixon AK, et al. Screening with magnetic resonance imaging and mammography of a UK population at high familial risk of breast cancer: a prospective multicentre cohort study (MARIBS). Lancet 2005;365:1769-1778

3. Warner E, Plewes DB, Hill KA, et al. Surveillance of BRCA1 and BRCA2 mutation carriers with magnetic resonance imaging, ultrasound, mammography, and clinical breast examination. JAMA 2004;292:13171325.
4. Saslow D, Boetes C, Burke W, et al. American Cancer Society guidelines for breast cancer screening with MRI as an adjunct to mammography. CA Cancer J Clin 2007;57:75-89.

5. Orel S. Who should have breast magnetic resonance imaging evaluation? JCO 2008;26:703-711.

6. Turnbill L, Brown S, Harvey I, et al. Comparative effectiveness of MRI in breast cancer (COMICE) trial: randomized controlled trial Lancet 2010:375:528-530.

7. Katipamula R, Degnim A, Hoskin T, et al. Trends in mastectomy rates at the Mayo Clinic Rochester: Effect of surgical year and preoperative magnetic resonance imaging. JCO 2009;27:4082-4088.

8. NIH consensus conference: Treatment of early-stage breast cancer. JAMA 1991;265:391-395.

9. Lazovich D, Solomon CC, Thomas DB, et al. Breast conservation therapy in the United States following the 1990 National Institutes of Health Consensus Development Conference on the treatment of patients with early stage invasive breast cancer. Cancer 1999;86: 628-637.

10. Wapnir IL, Anderson SJ, Mamounas EP, et al. Prognosis after ipsilateral breast cancer recurrence and ocoregional recurrences in five National Surgical Adjuvant Breast and Bowel Project node-positive adjuvant breast cancer trials. J Clin Oncol 2006;24:2028-2037.

11. Nauven PL, Taghian AG, Katz MS, et al. Breast cancer subtype approximated by oestrogen receptor progesterone receptor and HER2 is associated with local and distant recurrence after breast-conserving surgery. J Clin Oncol 2008;26:2373-2378.

12. Pettit K, Swatske M, Gao F, et al. The impact of breast MRI on surgical decision-making: are patients at risk for mastectomy? J Surg Oncol 2009;100:553-558.

13. Solin L, Orel S, Hwang W, et al. Relationship of breast magnetic resonance imaging to outcome after breast-conservation treatment with radiation for women with early-stage invasive breast cancer or ductal carcinoma in situ. JCO 2008;26:386-391.

14. King T, Saktr R, Patil S, et al. Clinical management factors contribute to the decision for contralateral prophylactic mastectomy. JCO 2011;29:2158-2164.

15. Early Breast Cancer Trialists' Collaborative Group (EBCTCG). Effects of chemotherapy and hormonal therapy for early breast cancer on recurrence and 15-year survival: an overview of the randomised trials. Lancet 2005;365:1687-1717. 